Research Article

\title{
Searching for Slip Surface of Disturbed Slope Based on Logistic Function
}

\author{
Yalin Zhu, ${ }^{1,3}$ Xuefeng Peng $\mathbb{D},{ }^{2}$ Kun Tan, ${ }^{1}$ Ming Shi, ${ }^{4}$ Yin Hong, ${ }^{1}$ and Yixian Wang $\mathbb{D}^{1}$ \\ ${ }^{1}$ School of Civil Engineering, Hefei University of Technology, Hefei, China \\ ${ }^{2}$ Anhui Zhongya Steel Structure Engineering Co., Ltd., Hefei, China \\ ${ }^{3}$ Anhui Key Laboratory of Civil Engineering Structures and Materials, Hefei, China \\ ${ }^{4}$ China Railway Hefei Institute of Architectural Municipal Engineering Design Co. Ltd., Hefei, China
}

Correspondence should be addressed to Xuefeng Peng; 2746031678@qq.com and Yixian Wang; wangyixian2012@hfut.edu.cn

Received 8 October 2019; Accepted 10 December 2019; Published 10 March 2020

Academic Editor: Mickaël Lallart

Copyright (C) 2020 Yalin Zhu et al. This is an open access article distributed under the Creative Commons Attribution License, which permits unrestricted use, distribution, and reproduction in any medium, provided the original work is properly cited.

\begin{abstract}
In the slope stability analysis, how to make the slip surface of the structure more precise and smoother has been the focus of research when fitting the slip surface with known numerical points. The study found that the logistic function has both advantages in fitting the slip surface. The related parameters $(M, A$, and $K)$ are derived by the threshold, symmetry, and precision control of its function. Logistic function constructs the slip surface and compares it with the broken-line slip surface; the numerical results show that the slip points on the two slip surfaces are consistent, indicating that the logistic function fits the curve correctly; the logistic function smooths the original polyline curve, which facilitates solving the direction vector of the curve.
\end{abstract}

\section{Introduction}

For the study of slope stability, it is important to solve two types of problems: the calculation of the safety factor and the search of the potential slip surface [1-3]. There are many ways to define the safety factor, and the two methods that engineers use the most are the limit balance method and the strength reduction method.

Among them, the limit equilibrium method is dominant [4]. It takes the rigid strip on the specific slip surface as the research object and performs static analysis to obtain the corresponding intensity storage value $[5,6]$. However, this method cannot calculate the deformation and displacement results of the structure, especially for the slide surfaces [7, 8]. With the development of computers, more scholars use numerical methods to calculate the stability of the slope $[9,10]$. This method can not only calculate the safety factor but also obtain the stress-strain field of the slope to solve the potential slip surface. For numerical analysis, the object of calculation is often the model unit, so when searching for the slip surface, it directly obtains the key coordinate point on the slip surface (referred to as the slip point) [11, 12].
In geotechnical engineering, a large number of observations show that the sliding surface of a slope is often similar to a cylindrical surface. Therefore, on the side section of the slope, the slip surface can be simplified into a curve [13-15]. This law provides a convenient way for slope stability analysis. It enables the analysis of landslides to be treated approximately as a plane problem, and the sliding surface is regarded as a sliding curve [16, 17]. A general polygonal sliding surface is constructed by using a linear function as a basis function [18]. These slip surfaces are continuous but not smooth enough. This can result in an inaccurate slope at the slip point, which makes it impossible to calculate the direction cosine [19]. Obviously, the unit jump function is not the only way to construct a slip surface. The most common is to use a polynomial to fit the curve because the general function can be expanded into a polynomial using Taylor series [20]. This method is to perform a certain mathematical function repair on the obtained curve. It interferes with the randomness of the slip surface and causes the loss of some slip points [21]. Taking into account the property of the unit jump function, the logistic function is used instead of it as a basis function [22]. 
This article discusses how the logistic function constructs a slip surface in a two-dimensional space and how to calculate related parameters.

\section{Sliding Surface Curve Based on the Logistic Function}

For any shape of the slip surface, people usually use a series of discrete points to construct [23]. From Figure 1(a), the two points are connected to form a polygonal line-shaped sliding surface.

This polygonal-type slip surface is mathematically modeled by a series of jump functions as a basis function. Deriving the slip planes gives their differential functions (Figure 1(b)). It is a series of step functions with unit step functions [24]:

$$
H(t)=\left\{\begin{array}{l}
1(t \geq 0), \\
0(t<0) .
\end{array}\right.
$$

Superimpose formula (1) to obtain the sliding surface differential function expression:

$$
g(x)=\sum_{i=1}^{n} a_{i} H\left(x-b_{i}\right)
$$

where $a_{i}$ is the slope difference, $b_{i}$ is the slip point coordinate, and $n$ is the number of calculation intervals.

Obviously, the function value at each slip point of Figure 1(b) is not unique. This function is used to obtain the slip surface curve by integrating, which will result in the inability to solve the direction cosine of each slip point.

2.1. Logistic Property Study. The logistic function is a sigmoid function named after Pierre Francois Velul in 1844 or 1845 when studying its relationship with population growth; its function expression is as follows $[25,26]$ :

$$
f(x)=\frac{M}{1+A e^{-K x}},
$$

where $M, A$, and $K$ are the basic parameters.

Deriving equation (3) leads to

$$
f^{\prime}(x)=K M \frac{A e^{-K x}}{\left(A e^{-K x}+1\right)^{2}} .
$$

Let $M=1, K=1$, and $A=1$ and get the corresponding logistic function and the curve graph of its derivative (Figure 2). It has three important characteristics: the logistic function is a threshold function, and the corresponding function value is $0<\operatorname{Logistic}(x)<1$; it has been proved that the logistic function in this graph is strictly symmetric about $(0,0.5)$; and the logistic function is a smooth curve.

Integrate equation (3) to get the following equation:

$$
Y(x)=\frac{M}{K}\left(\ln \left(A e^{-K x}+1\right)+x\right) \text {. }
$$

Figure 3 shows a comparison of the graph after logistic integration with the graph obtained by integrating the unit jump function $g(x)$. From Figure 3, the two function curves are consistent at the beginning and the end, which can ensure the accuracy of the smoothed surface. When $X=0$, the logistic curve is smoother at the turning point, and the unit jump function is connected by a broken line. From this, the logistic function can smooth the curve.

\subsection{The Composition of the Slip Surface Curve of the Logistic} Function. In order to facilitate the calculation, the sliding surface differential function needs to be divided into calculation intervals. The assumptions are as follows: there are $n+1$ slip points denoted by the corresponding abscissa: $u_{0}, u_{1}, u_{2}, \ldots, u_{n}$. Solve the slope between every two points denoted as $v_{1}, v_{2}, v_{3}, \ldots, v_{n}$. Taking the midpoint of the two slipping points as the critical point of the division, it is recorded as $b_{1}, b_{2}, b_{3}, \ldots, b_{n}$, and each interval $\left(b_{1} \sim b_{2}, b_{2} \sim b_{3}, \ldots, b_{n-1} \sim b_{n}\right)$ can be regarded as the object of research. As shown in Figure 4(a), the slip surface differential function in the figure has five slip points and is divided into fivesegment calculation intervals.

Each calculation interval is taken apart as a separate calculation object, and the initial coordinate $Y$ value is reset to zero, which makes each calculation interval a unit jump function, as shown in Figure 4(b).

The logistic function replaces the original unit jump function and translates $u$ units on the coordinate axis to coincide with the corresponding calculation interval. The logistic function of different intervals is obtained, which is denoted as $y_{i}$. The formula is

$$
y_{i}=\frac{M_{i}}{1+A_{i} e^{-K_{i}\left(x-u_{i}\right)}}
$$

where $M_{i}, A_{i}$, and $K_{i}$ are the function parameters of each interval segment, $i$ : $1 \sim n-1$.

Figure 5 shows the $y_{i}$ function graphs of different intervals. The $x$ values of the translation are 20,40, and 60, respectively.

The function of each calculation interval is accumulated to obtain a smoothed sliding surface curve differential function (as shown in Figure 6). The formula is

$$
z(x)=\sum_{i=1}^{n} y_{i}
$$

Substituting equation (6) in (7) and adding the slope initial value

$$
z=v_{0}+\sum_{i=1}^{n} \frac{M_{i}}{1+A_{i} e^{-K_{i}\left(x-u_{i}\right)}}
$$

where $v_{i}$ represents the slope of each calculation interval.

The differential function (equation (8)) is integrated to obtain the corresponding slip surface curve, as shown in Figure 7.

The formula is

$$
Z=v_{0} x+Y_{0}+\sum_{i=1}^{n} \frac{M_{i}}{K_{i}}\left(\ln \left(A_{i} e^{-K_{i}\left(x-u_{i}\right)}+1\right)+\left(x-u_{i}\right)\right) \text {, }
$$

where $y_{0}$ is the initial value of the $y$ value of the slip point. 


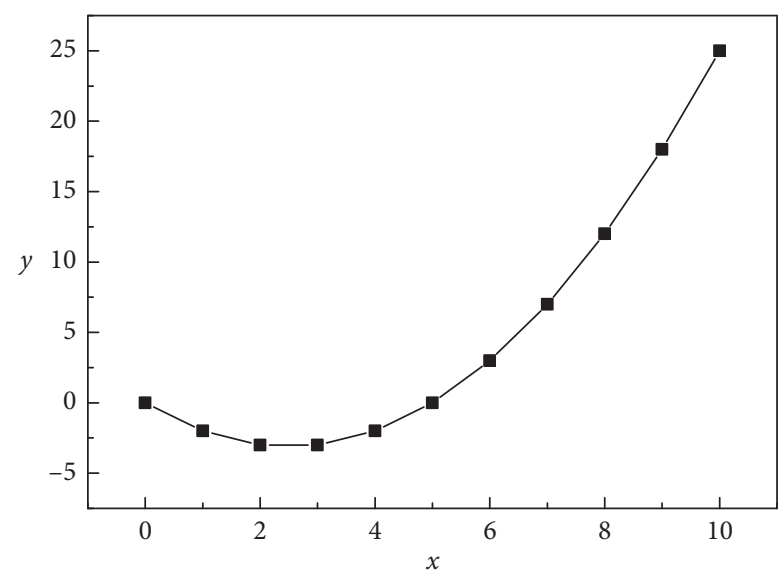

(a)

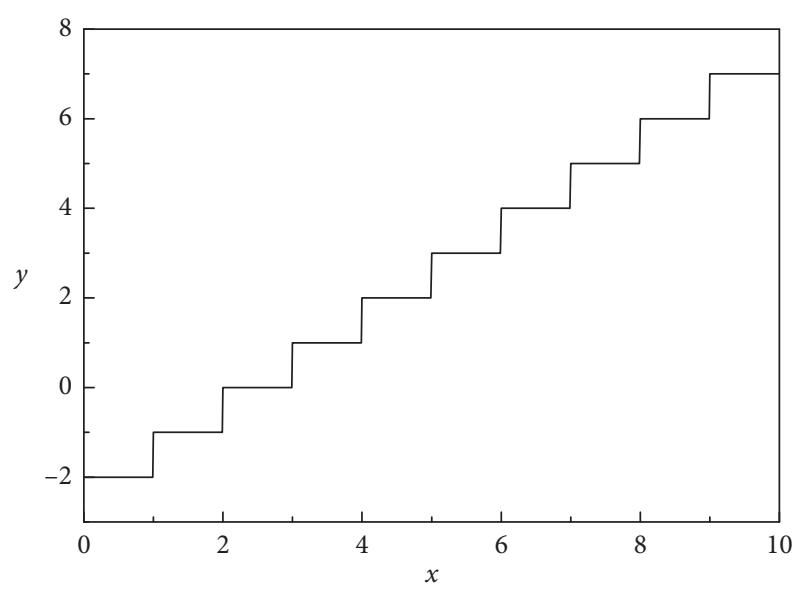

(b)

Figure 1: Polyline slip surface.

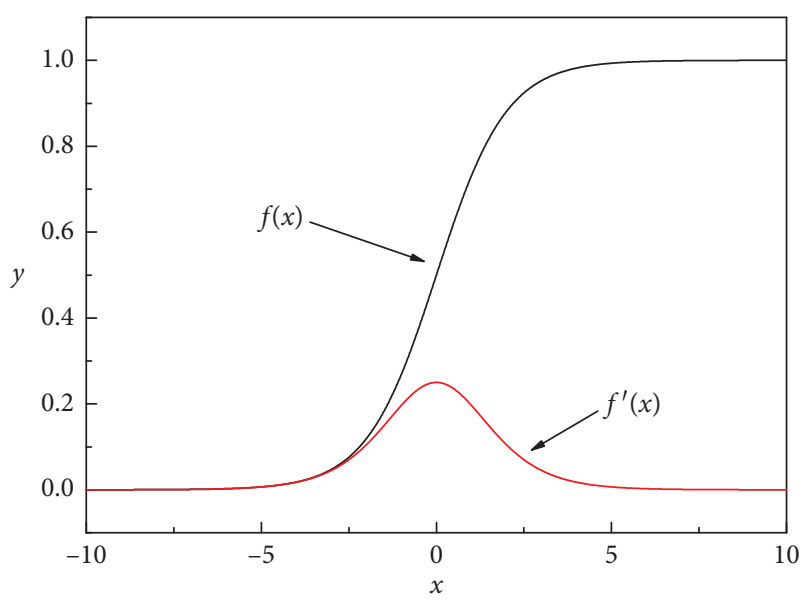

FIGURE 2: Logistic function and its derivative function.

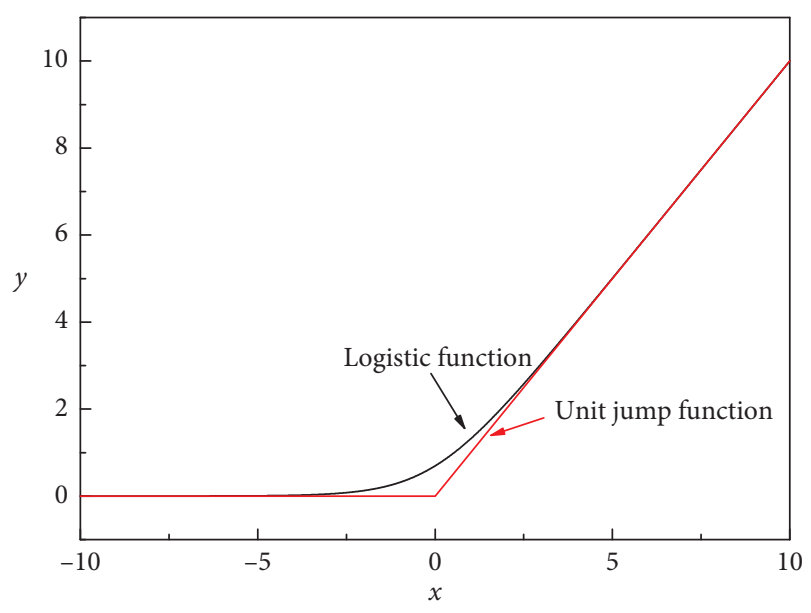

FIGURE 3: Function diagram comparison chart.

2.3. Logistic Parameters. The logistic function is mainly controlled by three parameters: $\mathrm{M}, \mathrm{K}$, and $\mathrm{A}$. In this section, the laws and properties of these three parameters are analyzed, and the calculation method for determining the parameters is obtained.

2.3.1. Parameter $M$. The slip surface differential function is superimposed by the logistic function, so the size of the $\mathrm{M}$ parameter is determined by the slope difference between each calculation interval by the following formula:

$$
M_{i}=v_{i+1}-v_{i} .
$$

Figure 8 shows a logistic function image with parameter $M$ of $1,2,3$, and 4, respectively (the other two parameters are all 1). As the parameter $\mathrm{M}$ increases, the upper limit of the logistic function increases continuously, and the parameter $\mathrm{M}$ controls the threshold size of the function.

2.3.2. Parameter A. The logistic function is a composite function in which the exponential function is constant over $(0,1)$ points. Then, the slip point $\left(u_{i}, y_{i}\right)$ is arbitrarily selected and brought into equation (6) to obtain

$$
\frac{M_{i}}{1+A_{i}}=y_{i} \text {. }
$$

Then, the deformation is available:

$$
A_{i}=\frac{M_{i}}{y_{i}}-1 \text {. }
$$

From the aforementioned equation, the parameter $A_{i}$ is determined by two variables, $M_{i}$ and $y_{i}$. Since the value of $M_{i}$ is a known condition, the key to the calculation of the parameter $A_{i}$ is to obtain the value of $Y_{i}$. Figure 9 shows that $A$ is the slip point on the differential equation curve before and after smoothing. In order to keep the value of the differential curve integrated, it is necessary to make the area around point $A$ equal, $S 1=\mathrm{S} 2$.

Because of the symmetry of the logistic function, let the ordinate of the $\mathrm{O}$ point be the average of $v_{i}$ and $v_{i+1}$, that is, 


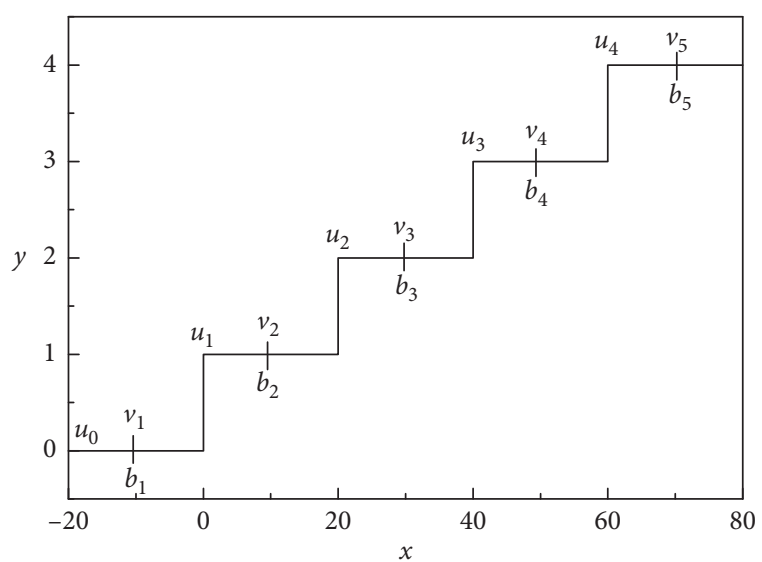

(a)

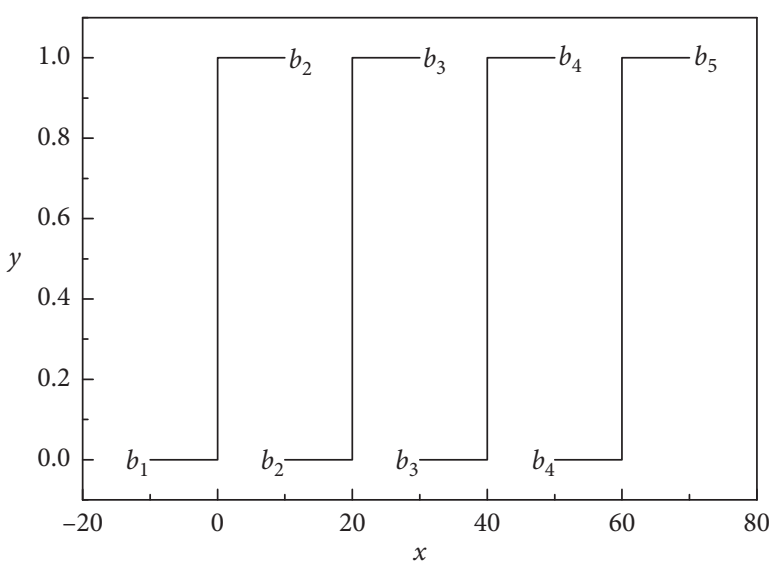

(b)

Figure 4: Schematic diagram of differential function interval division of the slip surface.

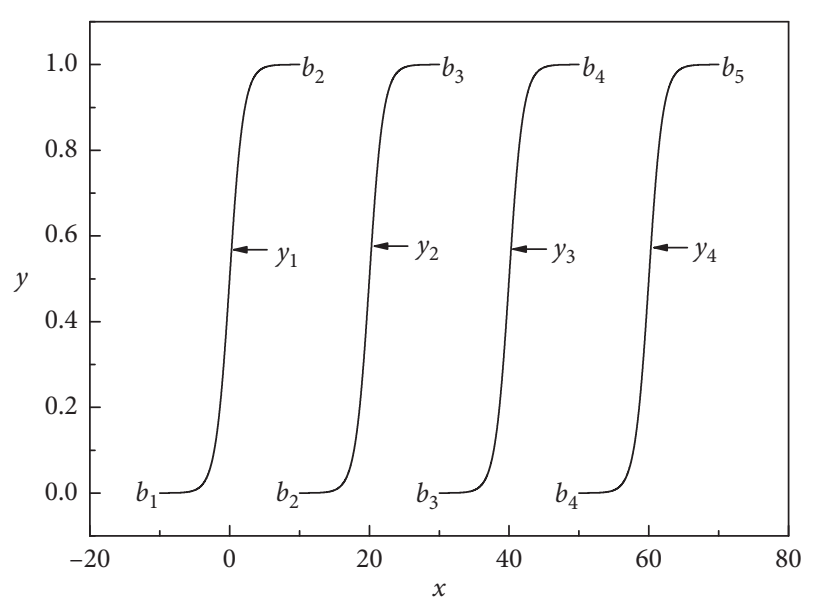

Figure 5: Diagram of the logistic function after translation.

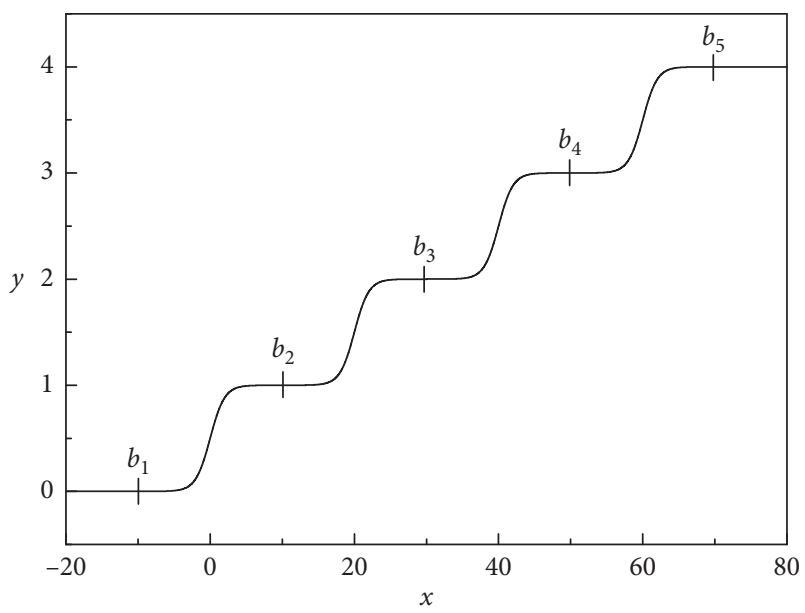

FIgURE 6: Logistic function synthesis diagram.

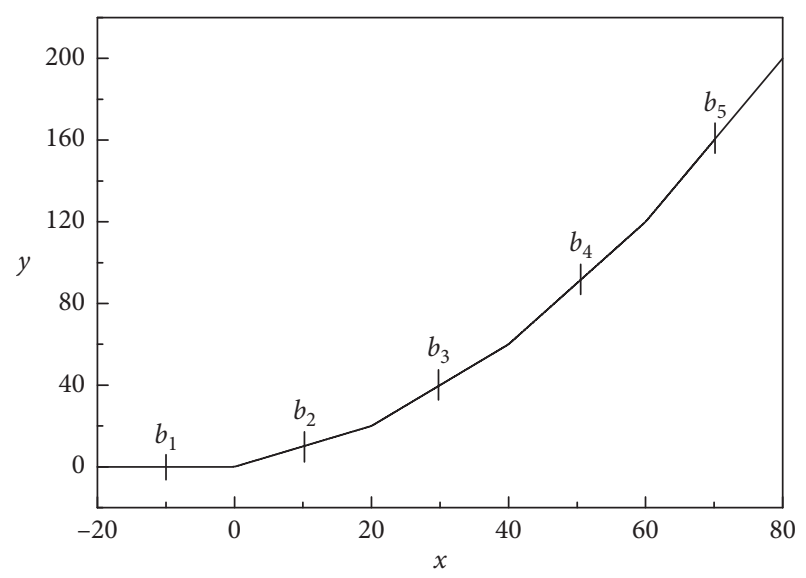

FIGURE 7: Schematic diagram of the slip surface based on the logistic function.

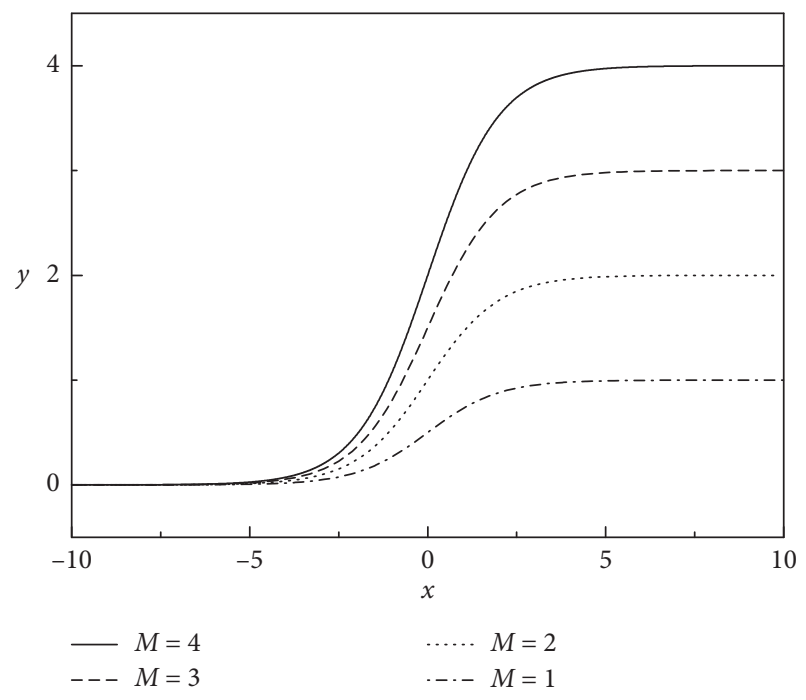

FIgURE 8: Comparison of different $M$ value function diagram. 


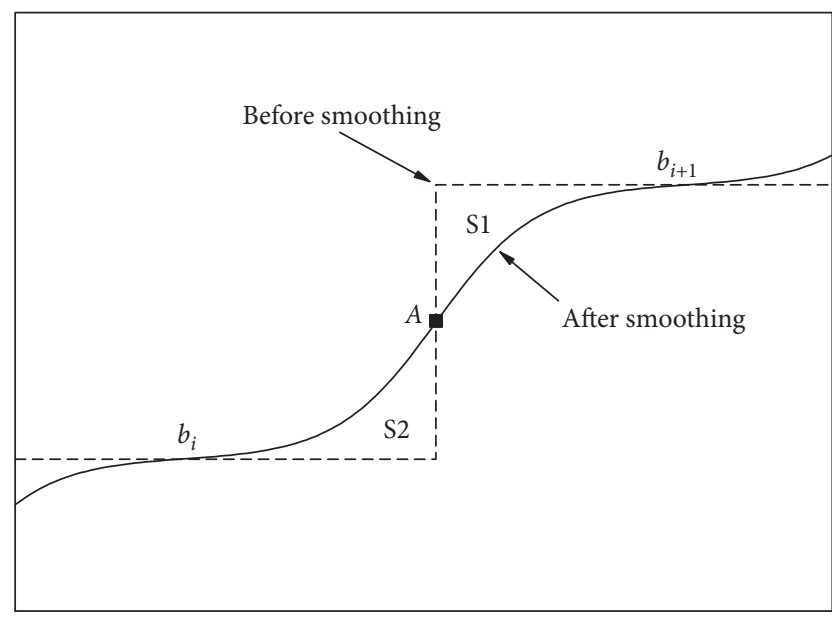

Figure 9: Comparison of differential equations.

$$
y_{i}=\frac{v_{i+1}-v_{i}}{2}
$$

Substituting (13) and (10) in (12),

$$
A_{i}=1 \text {. }
$$

When the parameter $A_{i}$ is a constant value of 1 , the logistic function can be made symmetric about $\left(u_{i}\right.$, $\left(v_{i}+v_{i+1}\right) / 2$.

2.3.3. Parameter $K$. The $K$ value mainly affects the speed at which the function value reaches the threshold. The $K$ value is a constant greater than 1 , and as the $K$ value increases, the speed at which the function changes in value is faster. As shown in Figure 10 , the $K$ value changes from 1 to 4 , and the number of corners of the curve is getting smaller.

The closer the $K$ value is to 1 , the smoother the curve, but the smoother the curve, the greater the deviation at the endpoint of the calculation. In Figure 10, the function with a larger value of $K$ has a larger deviation between the value of the point at both ends and the original value. When $x$ is at both ends $(x=-3$ or $x=3)$, the function of $K=4$ has the largest deviation from the original value $(y=0$ or $y=1)$. Figure 11 shows the deviation between the function values of the different $K$ values and the original values, where the left endpoint function value is compared to 0 and the right endpoint is compared to 1 . When $K=1$, the deviation generated is the largest, and the accuracy at this time cannot meet the requirements. If this differential function is integrated, it will cause a large deviation. When $K=2$, the deviation is already close to 0 , and the deviation becomes smaller and smaller as the value of $x$ increases.

From the above analysis, the accuracy of the endpoint needs to be controlled. For the convenience of calculation, this paper limits the error of the right endpoint of each calculation interval. Let any coordinate point on the right side of the slip point $u_{i}$ be $x_{i}$, at which point the function value reaches the target value. The formula is

$$
\lim _{x \rightarrow x_{i}} y_{i}(x)=v_{i+1}-v_{i}
$$

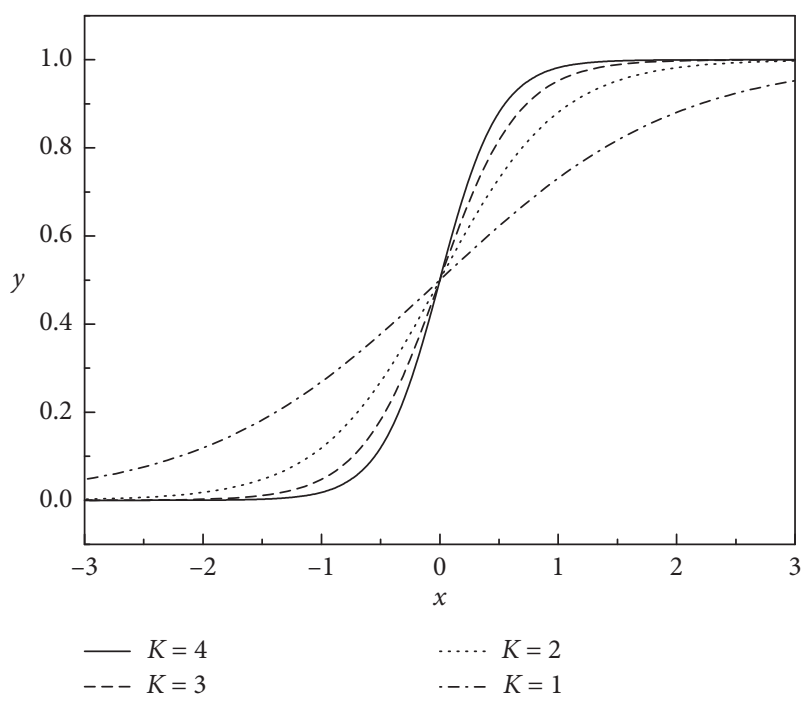

Figure 10: Diagram comparison of different $K$ value functions.

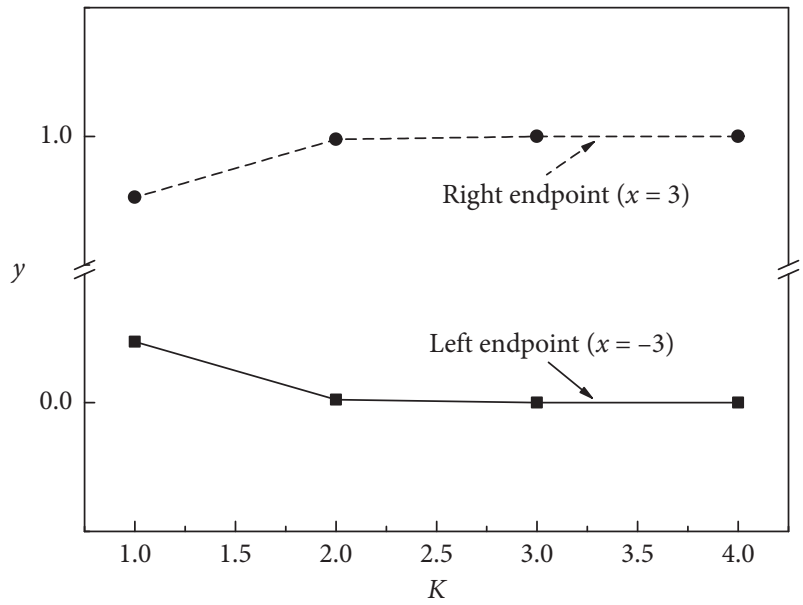

Figure 11: Deviation of different $K$ values at two endpoints.

Substituting formulas (6) and (10) in (15),

$$
\lim _{x \longrightarrow x_{i}} \frac{M_{i}}{1+A_{i} e^{-K_{i}\left(x-u_{i}\right)}}=M_{i} \text {. }
$$

Simplify:

$$
\lim _{x \longrightarrow x_{i}} e^{-K_{i}\left(x-u_{i}\right)}=0 .
$$

If the formula (17) is simplified, we get

$$
k_{i}=\lim _{x \longrightarrow x_{i}} \frac{\ln 0}{u_{i}-x} .
$$

With equation (18), the limit of the values of $K_{i}$ is inexistent when $(x) \longrightarrow\left(x_{i}\right)$. Therefore, let equation (17) be equal to any value that satisfies accuracy. In order to meet the accuracy requirements in engineering, this article assumes that the value is $1 \times 10^{-4}$ and

$$
K_{i}=\frac{9.21}{x_{i}-u_{i}} .
$$




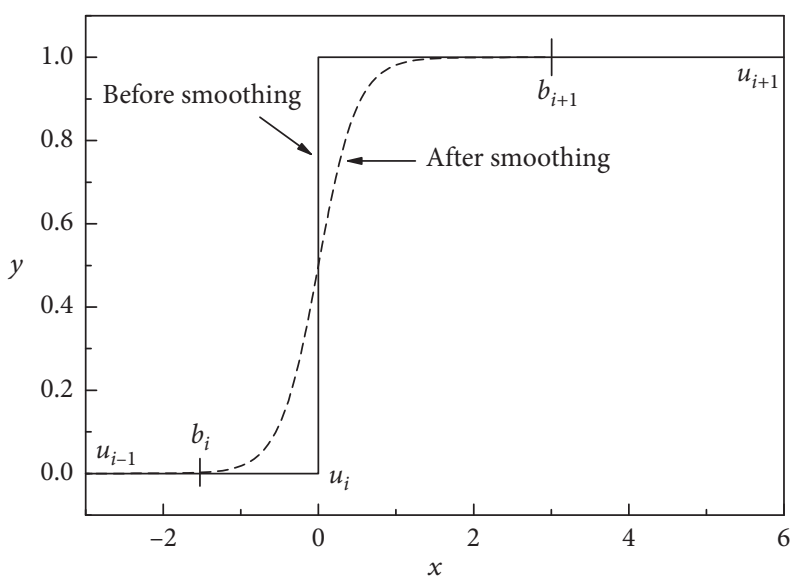

FIGURE 12: Calculation interval diagram.

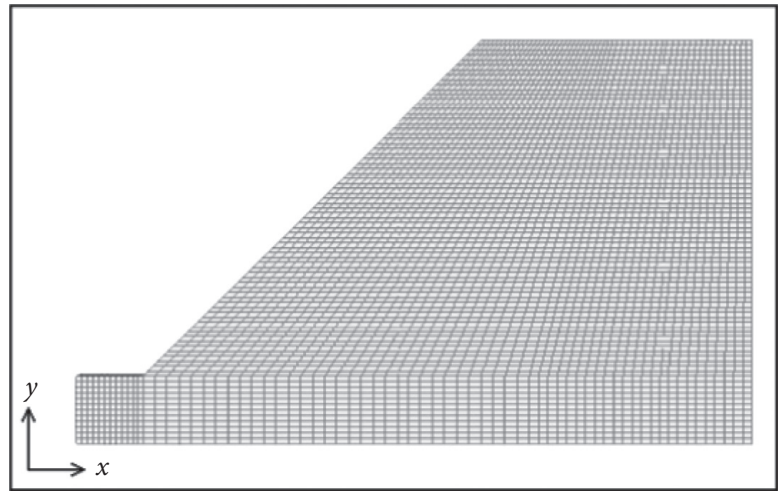

(a)

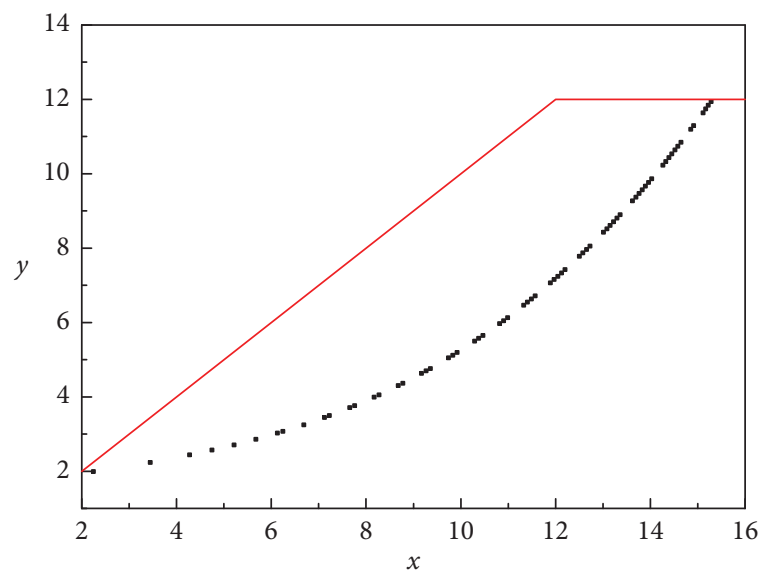

(b)

FIgURE 13: Slope slip diagram.

From the aforementioned equation, it is necessary to determine the value of $x_{i}$ to obtain the size of $K_{i}$. In order to ensure the accuracy after the integration, the value of the slip point to the point near the two ends is selected, and the value is equal to $x_{i}-u_{i}$.

Let $p_{i}=x_{i}-u_{i}$, and the formula is

$$
\left\{\begin{array}{l}
p_{i}=\min \left(\left|b_{i+1}-u_{i}\right|,\left|b_{i}-u_{i}\right|\right), \\
K_{i}=\frac{9.21}{p_{i}} .
\end{array}\right.
$$

From Figure 12, comparing the $b_{i} \sim u_{i}$ segment and the $b_{i+1} \sim \mathcal{u}_{i}$ segment in the graph, the length of the former is smaller than the latter, so the length of the $b_{i} \sim u_{i}$ segment is selected for calculation. If the calculation interval is a symmetric interval, select any endpoint.

\section{Case Verification}

The paper uses the maximum point of shear strain increment as the structural point of the slip surface of the slope [27].
When the slope is in a critical equilibrium state, the position of the potential slip surface coincides with the position of the maximum point of the shear strain increment, and the paper uses the strength reduction method to search for this state $[10,12]$.

The numerical analysis software used in the paper is FLAC3D, and Figure 13(a) shows a simplified diagram of the slope model $[28,29]$. There are many geotechnical constitutive models in FLAC3D software, the most widely used of which is the Mohr-Coulomb model. This model has two outstanding advantages: (a) this model is suitable for simulating loose or cemented granular materials; (b) this model is computationally efficient, which can save more memory and time; [30-32]. Therefore, Mohr-Coulomb model is adopted for the side slope, and the corresponding slope parameters are the slope height $\mathrm{H}: 10 \mathrm{~m}$, the slope ratio i: $1: 1$, the cohesion force c: $12.38 \mathrm{kPa}$, the internal friction angle $\varphi: 20^{\circ}$, the density: $1800 \mathrm{~kg} / \mathrm{m}^{-3}$, the shear modulus G: $10 \mathrm{GPa}$, and the bulk modulus $K$ : $30 \mathrm{GPa}$.

When the slope reaches a critical state, the safety factor is 1.05. The corresponding slope shear strain information is extracted, and the maximum value of the shear strain 


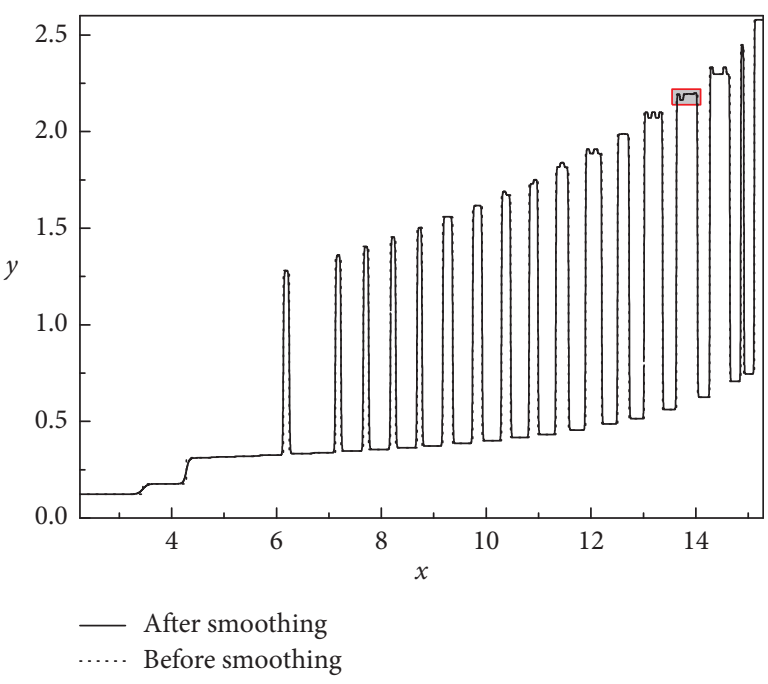

(a)

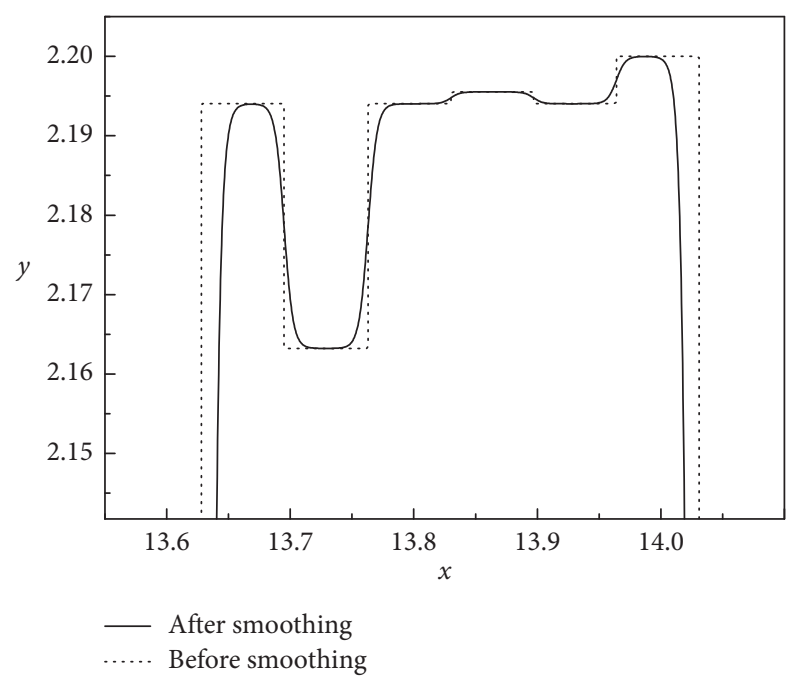

(b)

FIgURE 14: Sliding surface differential comparison diagram.

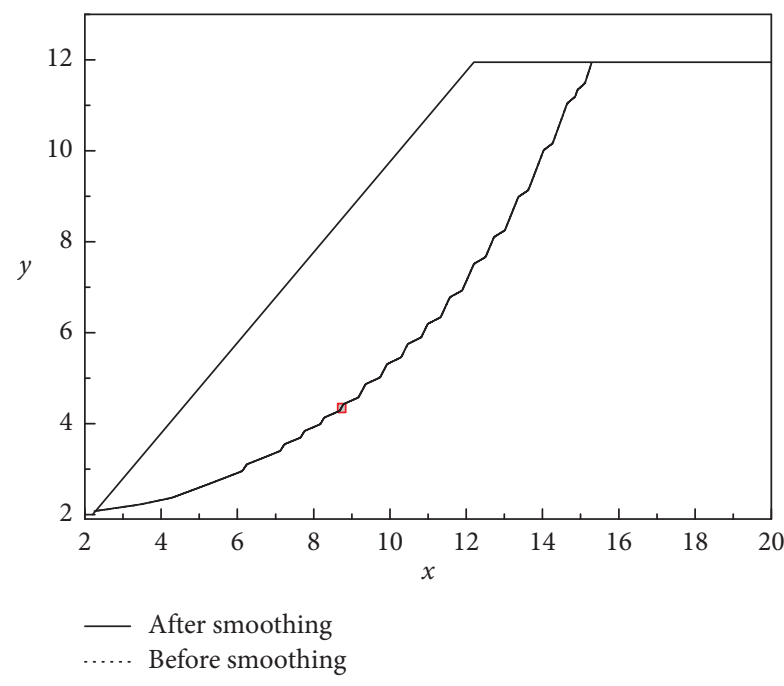

(a)

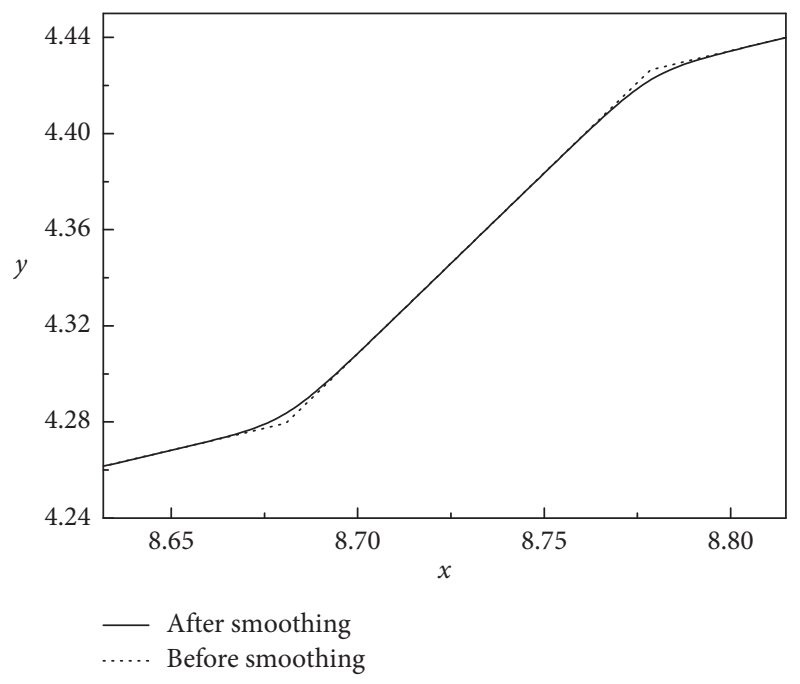

(b)

FIgURE 15: Slope slip surface curve comparison diagram.

increment of each layer is obtained by layer-by-layer search using MATLAB software [33]. Figure 13(b) shows the maximum value of the shear strain increment of each layer.

The obtained slip surface coordinates are brought into formulas (10), (14), and (19) to obtain the parameters $M_{i}, K_{i}$, and $A_{i}$ of the logistic function. The slip surface differential function can be obtained by using equation (8), and the corresponding graph is shown in Figure 14(a). From the figure, the curves before and after smoothing are completely consistent in value, indicating the correctness of the calculation. Also, for more clear observation, the red frame area in the figure is enlarged. Figure 14(b) shows the enlarged curve. It can be seen that the smoothed curve is smoother than the original curve. The curve consisting of logistic can be strictly symmetric about the original curve.
Using formula (9) to integrate the differential image of the slip surface, the curve of the slip surface can be obtained as shown in Figure 15(a). Similarly, the two curves are selected for amplification, see Figure 15(b). In the enlarged graph, the line segment connected by a smooth parabola at the slip point replaces the line segment originally connected by the polyline.

\section{Conclusions}

Through the mathematical analysis of the slip surface of the broken line, the logistic function is used to replace the original unit jump function, and the original polyline is smoothed. The three main parameters $(M, A$, and $K)$ of the logistic function are numerically analyzed, and the formulas 
of the three parameters are derived; the following conclusions are obtained:

(1) In addition to accurately reflect the change in slope, the logistic function can also smooth the turning points

(2) The properties of three important parameters $(M, A$, and $K$ ) in the logistic function are analyzed, and the corresponding calculation formula is derived

(3) Through the analysis of the example, it is proved that the constructed curve meets the exact requirements and achieves a good smoothing effect

\section{Data Availability}

All data included in this study are available from the corresponding author upon request.

\section{Conflicts of Interest}

The authors declare that they have no conflicts of interest.

\section{Acknowledgments}

This project was supported by the Natural Science Foundation of China (51409076 and 51774107), the Fundamental Research Funds for the Central Universities of China (PA2019GDPK0088), the Opening Project of the State Key Laboratory of Explosion Science and Technology, and Beijing Institute of Technology (KFJJ19-02M).

\section{References}

[1] H. Y. Ge, J. S. Tu, S. Xia, and J. G. Wang, "Inclined slice method for the calculation of slope stability safety factor," Advanced Materials Research, vol. 261-263, pp. 1459-1464, 2011.

[2] H. Lin and P. Cao, "Potential slip surfaces of slope with strength parameters," Advanced Materials Research, vol. 243-249, pp. 3315-3318, 2011.

[3] R. Chen, J. Liu, C. W. W. Ng, and Z. K. Chen, "Influence of slope angle on water flow in a three-layer capillary barrier soil cover under heavy rainfall," Soil Science Society of America Journal, vol. 83, no. 6, p. 1637, 2019.

[4] S. Y. Liu, L. T. Shao, and H. J. Li, "Slope stability analysis using the limit equilibrium method and two finite element methods," Computers and Geotechnics, vol. 63, pp. 291-298, 2015.

[5] A. W. Bishop and W. Alan, "The use of the slip circle in the stability analysis of slopes," Géotechnique, vol. 5, no. 1, pp. 7-17, 1955.

[6] M. D. James and Fellow, "State of the art: limit equilibrium and finite-element analysis of slopes," Journal of Geotechnical Engineering, vol. 122, no. 7, pp. 577-596, 1996.

[7] F. Laouafa and F. Darve, "Modelling of slope failure by a material instability mechanism," Computers and Geotechnics, vol. 29, no. 4, pp. 301-325, 2002.

[8] R. Chen, Y. Ge, Z. Chen, J. Liu, Y. Zhao, and Z. Li, “Analytical solution for one-dimensional contaminant diffusion through unsaturated soils beneath geomembrane," Journal of $\mathrm{Hy}$ drology, vol. 568, pp. 260-274, 2019.

[9] D. V. Griffiths and P. A. Lane, "Slope stability analysis by finite elements,” Geotechnique, vol. 49, no. 3, pp. 653-654, 1999.
[10] H. Zheng, D. F. Liu, and C. G. Li, "Slope stability analysis based on elasto-plastic finite element method," International Journal for Numerical Methods in Engineering, vol. 64, no. 14, pp. 1871-1888, 2005.

[11] Y. X. Wang, S. B. Shan, C. Zhang, and P. P. Guo, "Seismic response of tunnel lining structure in a thick expansive soil stratum," Tunnelling and Underground Space Technology, vol. 88, pp. 250-259, 2019.

[12] H. Zheng, L. G. Tham, and D. Liu, "On two definitions of the factor of safety commonly used in the finite element slope stability analysis," Computers and Geotechnics, vol. 33, no. 3, pp. 188-195, 2006.

[13] R. L. Michalowski, "Stability assessment of slopes with cracks using limit analysis," Canadian Geotechnical Journal, vol. 50, no. 10, pp. 1011-1021, 2013.

[14] H.-b. Du, F. Dai, Y. Xu, Z. Yan, and M.-d. Wei, "Mechanical responses and failure mechanism of hydrostatically pressurized rocks under combined compression-shear impacting," International Journal of Mechanical Sciences, vol. 165, Article ID 105219, 2020.

[15] A. Li, F. Dai, N. Xu, G. Gu, and Z. Hu, “Analysis of a complex flexural toppling failure of large underground caverns in layered rock masses," Rock Mechanics and Rock Engineering, vol. 52, no. 9, pp. 3157-3181, 2019.

[16] B. Li, T. Li, N. Xu, F. Dai, W. Chen, and Y. Tan, "Stability assessment of the left bank slope of the baihetan hydropower station, southwest China," International Journal of Rock Mechanics and Mining Sciences, vol. 104, pp. 34-44, 2018.

[17] B. Li, N. Xu, F. Dai, G. Zhang, and P. Xiao, "Dynamic analysis of rock mass deformation in large underground caverns considering microseismic data," International Journal of Rock Mechanics and Mining Sciences, vol. 122, Article ID 104078, 2019.

[18] X. F. Peng, Y. L. Zhu, and K. Tan, “Analysis of overburden layer thickness influence on dynamic response of concrete face rock-fill dam," Journal of Vibroengineering, vol. 21, no. 4, pp. 1116-1126, 2019.

[19] Y. M. Cheng, "Location of critical failure surface and some further studies on slope stability analysis," Computers and Geotechnics, vol. 30, no. 3, pp. 255-267, 2003.

[20] M. Hajiazizi and H. Tavana, "Determining three-dimensional non-spherical critical slip surface in earth slopes using an optimization method," Engineering Geology, vol. 153, pp. 114-124, 2013

[21] A. Cividini, "Application of numerical methods to slope stability analysis," Computational Mechanics-New Frontiers for the New Millennium, Elsevier, Amsterdam, Netherlands, pp. 323-332, 2001.

[22] D.-Y. Zhu, "A method for locating critical slip surfaces in slope stability analysis: reply," Canadian Geotechnical Journal, vol. 39, no. 3, pp. 771-337, 2002.

[23] Y. X. Jie, Z. K. Liu, and Z. H. Guo, "Mathematical functions for generating slip surfaces of slopes," Journal of Hydroelectric Engineering, vol. 37, pp. 108-120, 2018, in Chinese.

[24] A. Iliev, N. Kyurkchiev, and S. Markov, "On the approximation of the step function by some sigmoid functions," Mathematics and Computers in Simulation, vol. 133, pp. 223-234, 2017.

[25] G. Cybenko, "Approximation by superpositions of a sigmoidal function," Mathematics of Control, Signals, and Systems, vol. 2, no. 4, pp. 303-314, 1989.

[26] C.-X. Zhang, S. Xu, and J.-S. Zhang, "A novel variational Bayesian method for variable selection in logistic regression models," Computational Statistics \& Data Analysis, vol. 133, pp. 1-19, 2019. 
[27] L. Y. Cheng, J. Li, S. X. Chen, and X. J. Chu, "An application of the maximum shear strain increment in searching sliding surfaces," Applied Mechanics and Materials, vol. 423-426, pp. 1618-1622, 2013.

[28] S. Bock, "New open-source ansys-solidworks-flac3d geometry conversion programs," Journal of Sustainable Mining, vol. 14, no. 3, pp. 124-132, Article ID S2300396015301130, 2015.

[29] F. Wang and T. J. Cui, "The solution to the safety factor of vertical shaft enclosure with one-side elevation angle based on flac3d," Applied Mechanics and Materials, vol. 256-259, pp. 1303-1307, 2012.

[30] R. Wang, C. Li, J. Xu, and L. Pan, "Development and verification of large deformation model considering stiffness deterioration and shear dilation effect in FLAC3D," International Journal of Mining Science and Technology, vol. 28, no. 6, pp. 959-967, 2018.

[31] Y. Liu, F. Dai, L. Dong, N. Xu, and P. Feng, "Experimental investigation on the fatigue mechanical properties of intermittently jointed rock models under cyclic uniaxial compression with different loading parameters," Rock Mechanics and Rock Engineering, vol. 51, no. 1, pp. 47-68, 2018.

[32] Y. Xu and F. Dai, "Dynamic response and failure mechanism of brittle rocks under combined compression-shear loading experiments," Rock Mechanics and Rock Engineering, vol. 51, no. 3, pp. 747-764, 2018.

[33] Y. W. Kwon and H. C. Bang, The Finite Element Method Using Matlab, CRC Press, Boca Raton, FL, USA, 2015. 